\title{
Using multimodal pedagogies in writing centres to improve student writing
}

\author{
Arlene Archer \\ Writing Centre, University of Cape Town, South Africa \\ E-mail: arlene.archer@uct.ac.za
}

\begin{abstract}
This paper focuses on the affordances of multimodal pedagogies in writing centre environments to improve student writing. Writing centres have the potential to function as change agents, contributing towards changing the dominant attitudes to language and texts. Multimodal pedagogies encourage the use of a range of modes (such as talk, writing, music and images) and a range of resources (including multilingual, experiential, embodied and technologyenriched resources). This paper explores how consultants and students use a range of modes and ensembles of modes to develop thinking and learning in a multilingual and diverse higher education context. The dual role of consultants, being both 'reproducers' and 'interrogators' of academic discourse, is touched upon, and the importance of 'recognising' and drawing on the 'brought-along' resources in the training of consultants is highlighted. The unique nature of one-on-one consultations in the tertiary environment is explored, as well as the ways in which this pedagogical space can be enhanced through the use of multimodal pedagogies. To this end, the paper examines talk as an important mode in improving writing. It also interrogates working on the screen versus the page, the affordances of mind mapping, and the balancing of creativity and constraints in the writing consultation. The aim is to theorise a multimodal approach to improving student writing through the examination of practice. The contention is that multimodal pedagogies can acknowledge consultants and students as agentive, resourceful and creative meaning-makers. This is particularly relevant in a context in which autonomous and decontextualised models of student support persist and students continue to be constructed as 'lacking' in resources.
\end{abstract}

Keywords: higher education, multimodal pedagogies, one-on-one pedagogy, student writing, writing centres

\section{Introduction}

Almost 20 years ago, Prof Leon de Stadler encouraged me to initiate a writing centre at Stellenbosch University and helped me to develop the writing centre concept. He was interested in the relation between theory and practice, and it thus seems fitting that I write about this relationship in the domain of writing centres in a Festschrift dedicated to him. This paper focuses on the affordances of multimodal pedagogies in writing centres to improve student writing. 
Multimodal pedagogies recognise that "the use of modes in classrooms is always the effect of the work of culture, history and power in shaping materials into resources for meaning-making" and that pedagogic processes can be understood as "the selection and configuration of the semiotic resources available in the classroom" (Stein 2008:122). By mode, I mean a fully semiotically articulated means of representation and communication, such as language, image or music (Kress 2000:185). Learners engage with different modes in varying ways: they have different relationships, histories and competencies in relation to modes, including the use of digital technologies. In multimodal pedagogies, "there is a conscious awareness of the relationship between modes, learning and identity" (Stein 2008:122). Archer and Newfield (2014) have argued that multimodal pedagogies involve 'recognition' of students' broughtalong resources, including those resources not necessarily valued in higher education, such as multilingual, experiential, and embodied resources. Recognition is also about recognising student "interest" (Kress 2010) and agency, as people choose how to represent meaning from a range of possibilities which are shaped in a particular context. This recognition of students' resources is key to a transformative agenda in higher education.

A multimodal approach examines writing as part of a multimodal representational and communication landscape, and looks at the way in which writing is embedded within a wider semiotic frame in a social context. Important in writing centre practice is the exploration of how to define the scope, nature, and function of writing in higher education, especially when writing includes oral, visual, multimedia, and technology-enriched aspects. Digital media have enabled students to create and distribute multimodal work, which has implications for the ways we need to engage with both students and text. Many have argued that writing centres need to be equipped to assist with multimodal composition and argumentation (Huang and Archer 2017; Lee and Carpenter 2013) and to support multimodal communication using visual media such as videos (Pearman 2017), comics and MS PowerPoint (Huang 2015), medical pamphlets (Weiss 2014), posters and storyboards (Archer 2011), and information graphics (Prince and Archer 2014). Multimodal composition is about selecting semiotic resources, but it is also about the weight given to each mode in a particular text. One mode could, for instance, carry the proposition, and the other the evidence in an argument (Archer 2016). Pearman (2017) argues that in order for writing centres to respond to digital texts, effective training programmes should be devised to ensure that consultants can support these kinds of assignments. In particular, she looks at how video production can stimulate reflection and ideas about alternate ways of supporting writing. Pearman argues that, although digital assignments like video may not yet be common for higher education, "examining a non-traditional form of communication as well as how consultants can support its construction suggests a push in the range of capacities of writing centres" (2017:177).

This paper explores how consultants and students use a range of modes and ensembles of modes to extend and develop thinking and learning within multilingual and diverse higher education contexts. The hope is that this exploration will give us a better understanding of what multimodality has to offer as a pedagogic resource, and how it can be used to improve writing centre practice.

\section{Writing centres as transformative spaces}

It is important to recognise the value of unregulated spaces like writing centres where contesting knowledge and subject positions can be foregrounded, and where interrogation within and across disciplines can occur (Archer and Richards 2011). On the one hand, writing centre 
consultants could be understood as "reproducers" with a normalising function for "moral, intellectual and national sensibility" (Bernstein 2000:27). On the other hand, consultants could have the role of critically interrogating academic discourse and practices that may lead to changes in norms and standards. Here social, intellectual and personal boundaries are viewed "not as prisons, or stereotypes, but as tension points condensing the past and opening possible futures" (Bernstein 2000:25). One-on-one consultations can potentially develop "persons who both participate in the construction of the discourse and who are shaped by it" (Christie 1995:221). By 'discourse' I mean "sets of abstract principles which inform the way in which texts are coded by specific social groups, or within specific, institutional contexts", as defined by Kress and Van Leeuwen (2006:165). Making students aware of these textual codes and enabling them to employ them appropriately in the relevant contexts are the essential goals of a writing pedagogy that enables access for students. Because we recognise that meaning exists in many forms, "a new requirement is to develop means of recognition of different modes - and genres - through and in which learners / interpreters express the meanings which they have made" (Kress and Selander 2012:268). The explicit utilisation of multimodality in the one-onone consultation can enhance writing production and enable writers to explore actively different modes for constructing meaning.

Boughey and McKenna (2016) argue that the recent student protests at South African universities "have a great deal to do with the mastery of a 'way of being' required of students as they engage with higher education" (2016:2). Key to their argument is an understanding that reading and writing practices are profoundly social, involving the development of particular identities: "The unshifting dominance of an understanding of academic literacy practices as neutral and the concomitant construction of our students as decontextualized is implicated in these student protests" (2016:7). In this context, it is vital that we embrace potentially transformative spaces such as writing centres, and use the opportunities afforded by these spaces to interrogate the codes and practices of the academy, in relation to students' broughtalong resources, including student experience, multilingual resources, multimodal resources and embodied resources.

\section{Multimodal pedagogies of the training programme}

Writing centres are process-oriented spaces which welcome and foster multiple styles, processes and perspectives in writers (Bawarshi and Pelkowski 2003). Although writing centres differ across institutions in South Africa, most offer a walk-in, one-on-one consultancy service to students from all faculties and a range of academic levels. Most commonly, students bring a draft of their essay, which forms the basis of the consultation. The writing centre has no fixed agenda - there is no curriculum and no assessment - and contesting knowledge and subject positions can be foregrounded. Thus, writing centres have the potential to function as change agents, contributing towards changing the dominant attitudes to language and texts (Archer and Richards 2011).

\subsection{Drawing on consultants' multimodal resources: Objects that signify a relationship to writing}

Consultants at the University of Cape Town (UCT) Writing Centre are postgraduate students from a range of disciplines and they undergo initial training and ongoing training throughout the year. In order to exemplify ways of drawing on consultants' multimodal resources, I want to examine a warm-up activity used in the training programme. I did an exercise to get the 
consultants to reflect on their writing processes from a personal perspective. I tried to open up the conversation to allow them to draw on their own resources by getting them to reflect on an object that signifies their relationship to writing. By doing this, I hoped to find innovative ways of encouraging consultants to talk about themselves and their communities, using objects as metaphors to elicit indirect accounts of personal experience. In this multimodal approach to pedagogy, objects are seen as "reflections of the wider lives of communities and individuals" (Emmison and Smith 2000:111). While new meanings are created through 'recontextualization' (Bernstein 1996; Iedema 2003; Kell 2006), familiar objects are 'made strange' by examining them in new contexts, and objects can become catalysts for talking about experience in the training arena.

Some of the objects that the consultants identified were more obviously associated with writing, such as keyboards, pencils, paper and paintbrushes. According to one of the consultants, Anne, ${ }^{1}$ a pencil was "always quite short, chewed up at one end, eraser used up or broken off, very much worse for the wear and ugly". For her, writing was a process that was "difficult (note short eraser), stressful (bite marks), slow (pencil rather than computer), reassuring and old fashioned, personal, requires constant breaks (to sharpen pencil)". For another consultant, Matt, a blank page was seen as "possibility and a space to write", like a promise of change and exploration, but also like a mirror. A further consultant, Jo, saw a paintbrush as symbolising "the relationship in my mind between image and text, and the leap I made at a certain point when I conceived of writing as 'painting with words' - this began in poetry writing but now permeates my attitude to writing in all its forms". Then there was Maleson's rather cleverly chosen object, Prestik, a rubber-like, temporary and reusable adhesive. Maleson chose Prestik because of its elasticity, pliancy and capacity to change shape, which was analogous to the experimentation with form or genres in writing. Prestik also enabled you to pin things up, thus displaying aspects of yourself through writing. According to him, Prestik also expired and the pin-ups fell down after some time, requiring you to put up new ones and to "re-energise" the Prestik. Lastly, he commented that Prestik looks edible, but you cannot actually eat it. This revealed his relish for certain kinds of writing which he regarded as "quite delicious".

Other objects were more particular to the individuals' sociocultural milieu. Thandi spoke about a woven basket as an object that signified writing for her. The basket is made up of individual strands of reed which have little use on their own as they are fragile and not particularly attractive. This changes when they become the 'stuff' of the basket, which is not only beautiful and logical, but also useful. They can be of various sizes, shapes, uses and colours. Thandi saw basket weaving and writing as a social activity, but also as an individual activity: there can be single or multiple authors. She drew analogies between weaving and cohesion in writing, and pointed out the patience involved in both.

This exercise in the UCT Writing Centre training programme drew on the student consultants' cultural and personal resources. The use of objects as metaphors served to draw on the consultants' resources in order to enable a highly personal sharing of the common experience of writing. It also enabled the consultants to think through some of the complexities of writing and to develop a kind of 'home-grown' metalanguage which can be used in reflecting on writing processes within the community of consultants, and also within one-on-one consultations.

1 Pseudonyms are used. 
Another multimodal way of doing this during the course of training is to utilise strategies such as role-play.

\subsection{Use of role-play in the training programme}

During UCT Writing Centre training, the consultants act out mock consultations with each other. One participant acts as the student and the other as the consultant, and these roles are then reversed. Sometimes, a scenario is given to the student to act out; for instance, the 'angry student' or the 'proud student'. Role-play can be used as a serious learning activity with specific learning objectives, while at the same time remaining fun. In acting out the scenario, the consultants need to become aware of their body and voice in relation to the student, in aspects such as gesture, intonation, proximity, and positioning of the document under discussion. This is in addition to setting a consultation agenda, practising on-the-spot textual analysis, engaging the student in questions, eliciting responses, creating dialogue, and maintaining the focus on the task. These are complex operations and would not yield the same level of discussion and reflection without the experiential aspect of role-play. In order to exploit the full value of roleplay, it should be followed by a de-briefing or a shared reflection.

There are other ways of engaging the multimodal competencies of consultants, and specifically the body. For instance, in a similar performative exercise to role-play, Hunma (2016) reports on a study in which international students access their 'authorial self' by participating in a class using 'image theatre'. The students construct images by creating 'human sculptures' using their bodies, through which they present the challenges of their university contexts. They then create a sculpture of the ideal situation and visualise possibilities of getting from the 'now' to the 'ideal'. Hunma argues that this exercise can be explored as a valuable form of pre-writing, but can also be used to reflect on the authorial self in academic writing. According to Hunma (2016:189), "[t]heatre can become an interactive, multimodal option allowing teachers of writing to open up new performative sites for their students to express different aspects of their writer selves". In image theatre and in role-play, students and consultants are invited to negotiate the "positional and spatial boundaries of pedagogical spaces" and the "rules and reach for creative and critical textual performance" (Hunma 2016:189).

Having discussed some possibilities for multimodal pedagogies in the training of writing centre consultants, I now move on to look at ways of enhancing the one-on-one consultation utilising multimodal pedagogies.

\section{One-on-one pedagogy as multimodal event}

It has been widely acknowledged that the best way to learn to write is not in a traditional large classroom, but in a student-centred environment where students can explore and experiment with writing and get assistance from a critical but supportive audience in one-on-one consultations. The consultant guides students' thoughts and writing processes by questioning and clarifying, so that students learn to convey their meaning more effectively, and feel confident to be able to enter the academic debate. Actively employing the opportunities of multimodality allows writers to use extra-linguistic structures in the process of conceptualising meaning. Also, multimodal interventions can enable a range of diverse students' resources to emerge, including multilingual resources. Some multimodal interventions in the one-on-one consultation could include physically cutting up the essay and rearranging the parts; using images, mind maps and tables during discussion or in order to prompt discussion; various kinds 
of role-play in the consultation; talking about ideas (in any language); reading out loud; practising circumscribed writing within particular constraints; employing free writing; and moving between screen and page during the consultation. Owing to space constraints, I have considered only a few of these interventions, beginning with the mode of 'talk'.

\subsection{Talk as a mode used in improving writing}

One of the underlying premises of writing centre pedagogy is that a critical way of being develops through discussion and argument. Writing centres thus embrace the complex relationship between the spoken and the written, and how the written is understood by a reader. In order to encourage student independence, consultants offer students the opportunity to articulate problems and to explain what they are doing. The consultant's task is to help students to acquire a vocabulary or 'metalanguage' that allows them to draw back from what they have written, to talk about their writing, and to develop self-critical practices in order to appraise their work as they progress. At the end of a consultation, consultants often get the students to articulate the ways in which they felt the consultation had helped them. This is an important metacognitive activity as it helps students to become conscious of and to verbalise aspects of their own writing. This verbalisation on the part of the student is also often a good indicator to the consultant of how much learning has taken place.

It is useful for students to recognise that writing is not just a question of putting into physical form the words they say. Talking is a valuable form of pre-writing, but writing is not simply a recording of talk. Despite crossovers and borrowings, there are differences between oral and written modes. Written language does different things in different social contexts and for different social purposes, and uses a different linguistic technology than spoken language. With writing, the audience is usually absent (except in some online environments). Writing thus often needs to provide its own context. Talk, on the other hand, often depends on the environment, and the listener is usually present. One way of embracing multilingualism and enabling conceptual understanding is to invite students to talk about their writing needs in a language with which they feel comfortable, before writing in the medium of instruction.

Moving from the modes of talk to writing, writing to talk, images to talk to writing, talk to images to writing, involves a cognitive process which Kress (2010) calls "transduction". Transduction entails "re-articulation of meaning from the entities of one mode into the entities of the new mode" (Kress 2010:125). There is a move from one mode to another: from speech to writing, or from image or gesture to writing. In this move, there are pedagogical affordances. For instance, having students read their own essays aloud in a one-on-one consultation ensures student participation and avoids one-way communication. Students also often pick up on their own language mistakes and logical inconsistencies when reading aloud, and aspects such as punctuation become embodied through pacing as breathing. In the process that Kress (2010) calls 'transformation', the movement of meaning involves no change in mode (for instance, writing on the page and then the screen). Rather, transformation refers to the process of meaning change, through the re-ordering of the elements in a text (Kress 2010:129). The movement of meaning across genres or media in the process of transformation could change the logic of the meaning. It is clear that both aspects are of importance in writing, as we have to consider issues of transduction from one mode to another, and also the positioning of modes in relation to each other. This is also pertinent in relation to movements across media, such as from the screen to the page, or working in an online environment. 


\subsection{Screen versus page in a multimodal consultation}

Certain different texts support particular types of interaction, and materiality is thus a crucial component of multimodal genres and pedagogies. For instance, we can flip through a book by turning the pages, whereas in a screen-based document we need to scroll or click on hyperlinks. These artefacts thus offer different affordances for interaction (Bateman 2008:11). Jewitt (2006) has argued that the default shape of page and screen are different - the page is portrait, whereas the screen is landscape. In addition, the visual impact of page and screen is different; the historical associations and the arrangement of information are different. New understanding of space and time requires us to learn new strategies and consultation techniques that work in online environments. The online space predominantly employs verbal and visual modes, whereas face-to-face encounters offer a more tangible physical environment and embodied interaction. An online environment is rich with written text and often includes feedback only in the written mode. This can be seen as an advantage, as consultants are able to provide extensive written comments on student writing through writing questions, comments and suggestions. They need to be more explicit about the feedback than in face-to-face meetings. Tutoring in online spaces thus places "the burden on writing instead of personality" (Coogan in Kastman Breuch and Racine 2000:240).

Online writing consultations can either be synchronous, where students and consultants communicate in real time, or asynchronous, where students communicate in a delayed way such as via e-mail (Hyland and Hyland 2006:93). In terms of multimodal approaches to the one-onone consultation, it is worth considering the affordances of working on the screen versus on the page. When working on the screen, the student will find the text more immediate and perhaps more malleable. The student can make changes then and there. However, working with a document on the screen or in an online environment could lead to a focus on the product rather than on the process. It is difficult, for instance, to work on structure online and the danger may lie in resorting to 'editing' the student's document. The environment of the screen may be better for doing a task analysis, for example when one needs to organise a spatial display of information in malleable ways. The 'comments' function may be less intrusive than track changes, and feedback online can operate as a gateway to other sites through links. The online environment enables the easy dissemination, sharing and revising of texts, and includes opportunities for more collaborative forms of writing, such as the track changes function or 'google docs'.

Hyland and Hyland (2006) quote a study by Schultz (2000), which showed that students made more specific, local changes online and more global changes in the face-to-face encounter. This seemed to be because the online revisers were able to save and follow the detailed suggestions made in writing, while the face-to-face writers' global changes were facilitated by the more rapid back-and-forth interaction. It is clear that, overall, the online space presents different experiences for consultants in terms of forming relationships with online clients, procedures for responding to documents online, and creating appropriate tutor roles (Kastman Breuch and Racine 2000:246).

\subsection{Mind mapping}

Another useful multimodal scaffolding activity for writing that we employ in one-on-one consultations is that of mind mapping. A mind mapping process in the one-on-one consultation could enable a collaborative and negotiated approach to meaning making, where knowledge is 
co-constructed. Although many students may be familiar with brainstorming activities, they may not consider using mind maps in gathering and negotiating information, or planning assignments. However, mind mapping can be regarded as a crucial part of exploratory meaning making, a creative activity emerging as a material artefact. As a physical and mental process, it affords writers opportunities to "creatively generate, challenge and negotiate ideas, notions and questions of interest and then to compose these into meaningful arrangements" (Grant 2012). Decisions that may be discussed in the consultation include what topic headings and subheadings to choose and how to arrange and display these visually across or down a screen (if online) or on a sheet of newsprint.

According to Kress (2010), mind or concept maps attempt to 'fix' meaning in habitual ways and shape what viewers have come to recognise and expect of this type of visual-verbal display. He contends that a concept map is "organized through the affordances of image, using the semiotic logic of space and the modal affordance of spatial relations between simultaneously present entities" (Kress 2010:95). Visual semiotic resources such as shapes, colours, icons, arrows and lines may serve to engage participants "to do different kinds of semiotic work" (Bezemer and Kress 2008:171). According to Grant (2012), this inclusion of lexical and graphic modal resources and the spatial arrangement between them could encourage a 'consider all factors' cognitive/affective engagement, which may amplify creative brainstorming and innovative meaning making. The practice of mind mapping allows students to explore and construct a knowledge base by generating ideas that foreground their topic of interest and then enables them to arrange these ideas in hierarchical relationships. How students portray these relationships and explore the issues that arise as they grapple with choices around discourses, genres and modes are all the subject matter of one-on-one consultations.

\subsection{Exploring constraints and risk through artificial restrictions}

Another exercise to consider within the one-on-one consultation is to (co)create texts with artificial constraints, such as asking students to express the aim of their thesis in just one sentence; to frame their research as a story beginning 'once upon a time'; to describe an object that illuminates their research; and to freewrite for a fixed time period. Bell (2016) looks at the creative affordances for students when creating texts with artificial constraints, such as writing in particular visual forms. His projects emphasise the visual aspect of words, and it is the images or the layout that 'dictate' the writing, not the other way around. Within these constraints, students are able to make choices about the written text, the imagery, how to use space, the redistribution of words within tight limits, the coherence of the text. Paradoxically more weight is given to each word by using so few: "the more restrictions imposed ... the more important, difficult and rewarding word choice becomes. This sharpens the focus on the words, making them work harder to produce meaning" (Bell 2016:141).

Bell argues that he opened up the relationship between content and form by "forcing the students out of genre-sanctioned comfort zones, in order to foster risk-taking" (2016:150). This idea of 'risk' is useful to explore in a writing centre. Writing involves risk: "as we write, we always hover on the edge of commitment" (Chihota and Thesen 2014:133). Risk can include the sense of loss that writers and researchers experience when producing a written account of their research: "In the process of writing, various experiences and modes of expression are revised or erased along the way" (Thesen 2014:1). Thesen (2014) explores the relation between writing, risk and voice, particularly at postgraduate level in higher education and argues that risk taking can be utilised as a productive force for engaging in teaching, writing and knowledge 
production. There can be risk in producing multimodal texts, as well as in engaging in multimodal pedagogies in the writing centre. But, this risk can be generative as is demonstrated in the tasks which require writing under strict constraints, which ironically enable a kind of freedom and risk taking not appropriate in more conventional genres in academia.

\section{Implications for research and pedagogy in writing centres}

This paper has explored the affordances of multimodal pedagogies in writing centres to improve student writing, focusing on the training of consultants as well as the unique pedagogical potentials of one-on-one consultations, when including a range of verbal and visual modes and media. One of the implications of a multimodal perspective for research and pedagogy in writing centres includes the need to explore the affordances of modes as a vital part of developing academic literacies practices. More could be said about the ways in which new technologies enable modes to be configured and to circulate in different ways. To do this, we need a broader understanding of writing where meaning making, the role of agency, and signs of learning become central. To this end, I have argued that multimodal pedagogies in the writing centre environment can acknowledge both consultants and students as agentive, resourceful and creative meaning makers.

The multimodal strategies described here are used to promote metacognitive thinking through a range of pre-writing and writing activities in one-on-one consultations. There is also scope for writing centres to work collaboratively with subject specialists in the disciplines to integrate multimodal pedagogies and students' brought-along resources within a transformative higher education agenda. Writing centres could play a vital role in shifting assessment practices to include oral, visual, multimedia and technology-enriched aspects. To this end, it is important to develop metalanguages to facilitate awareness and analysis of multimodal textual constructions (e.g. Huang and Archer 2017). Multimodal communication can be used to promote writing development for normative and transformative purposes. This is particularly relevant in a context in which autonomous and decontextualised models of student support persist and students continue to be constructed as 'lacking' resources.

\section{Conclusion}

Jewitt has argued that in South Africa the connection between "research, educational practice, and an agenda for social and political change" (2014:xvi) is emphasised and that multimodal approaches in this context tend to pay attention to equity, participation and social justice. There is huge diversity in terms of language, culture and educational preparedness within the student population in most South African tertiary institutions. The one-on-one consultation as the basic teaching method of writing centres is premised on the belief that knowledge is not just something we acquire from books and teachers, it is something that is created by a community of knowledgeable peers. By exploring the possibilities of multimodal approaches in one-on-one consultations, we could harness the intellectual and creative potentials of learners from diverse social, intellectual and cultural environments. "The expanded forms of social and material practices associated with multimodal design can thus provide a platform for marginalized students to create complex texts" (Anderson, Stewart and Kachorsky 2017:111). Multimodality in writing centres can thus help to bring out the richness of diversity, in order to enable more people to participate in successful communication processes in different contexts. 


\section{References}

Anderson, K., O. Stewart and D. Kachorsky. 2017. Seeing academically marginalized students' multimodal designs from a position of strength. Written Communication 34(2): 104-134.

Archer, A. 2011. Clip-art or design: Exploring the challenges of multimodal texts for writing centres in higher education. Southern African Linguistics and Applied Language Studies 29(4): 387-399.

Archer, A. 2016. Multimodal academic argument: Ways of organizing knowledge across writing and image. In A. Archer and E. Breuer (eds.) Multimodality in higher education. G. Rijlaarsdam and T. Olive (series eds.) Studies in Writing, Vol. 33. Leiden: Brill. pp. 93-113.

Archer, A. and D. Newfield. 2014. Challenges and opportunities of multimodal approaches to education in South Africa. In A. Archer and D. Newfield (eds.) Multimodal approaches to research and pedagogy: Recognition, resources and access. Oxon and New York: Routledge. pp. 1-18.

Archer, A. and R. Richards. 2011. South African writing centres as alternate pedagogical spaces. In A. Archer and R. Richards (eds.) Changing spaces: Writing centres and access to higher education in South Africa. Stellenbosch: Sun Media. pp. 5-15.

Bateman, J.A. 2008. Multimodality and genre. A foundation for the systematic analysis of multimodal documents. New York: Palgrave MacMillan.

Bawarshi, A. and S. Pelkowski. 2003. Postcolonialism and the idea of a writing centre. In J. Murphy and S. Sherwood (eds.) St Martin's sourcebook for writing tutors. 2nd ed. Boston: Bedford/St Martin's.

Bell, S. 2016. Writing against formal constraints in art and design: Making words count. In A. Archer and E. Breuer (eds.) Multimodality in higher education. G. Rijlaarsdam and T. Olive (series eds.) Studies in Writing, Vol. 33. Leiden: Brill. pp. 136-166.

Bernstein, B. 1996. Pedagogy, symbolic control and identity. Theory, research, critique. London: Taylor and Francis.

Bernstein, B. 2000. Pedagogy, symbolic control and identity. 2nd rev ed. Oxford: Rowman and Littlefield.

Bezemer, J. and G. Kress. 2008. Writing in multimodal texts. A social semiotic account of designs for learning. Written Communication 25(2): 166-195.

Boughey, C. and S. McKenna. 2016. Academic literacy and the decontextualised learner. Critical Studies in Teaching and Learning 4(2): 1-9.

Chihota, C. and L. Thesen. 2014. Rehearsing 'the postgraduate condition' in writers' circles. In L. Thesen and L. Cooper (eds.) Risk in academic writing. Postgraduate students, their teachers and the making of knowledge. Bristol, Buffalo and Toronto: Multilingual Matters. 131-147.

Christie, F. 1995. Pedagogic discourse in the primary school. Linguistics and Education 7(3): 221-242.

Emmison, M. and P. Smith. 2000. Researching the visual. London, Thousand Oaks and New Delhi: SAGE Publications.

Grant, T. 2012. Scenario pedagogy as a participative teaching-learning approach to developing professional communication practices in higher education. Unpublished $\mathrm{PhD}$ dissertation, University of Cape Town, South Africa. 
Huang, C-W. 2015. Argument as design: A multimodal approach to academic argument in a digital age. Unpublished PhD dissertation, University of Cape Town, South Africa.

Huang, C-W. and A. Archer. 2017. Training writing centre tutors for argument in a digital age. In S. Clarence and L. Dison (eds.) Writing centres in higher education: Working in and across the disciplines. Stellenbosch: Sun Media. pp. 81-96.

Hunma, A. 2016. Multimodality in higher education. In A. Archer and E. Breuer (eds.) Multimodality in higher education. G. Rijlaarsdam and T. Olive (series eds.) Studies in Writing, Vol. 33. Leiden: Brill. 167-191.

Hyland, K. and F. Hyland. 2006. Feedback on second language students' writing. Language Teaching 39(2): 83-101.

Iedema, R. 2003. Multimodality, resemiotization: Extending the analysis of discourse as multi-semiotic practice. Visual Communication 2(1): 29-57.

Jewitt, C. 2006. Technology, literacy and learning. A multimodal approach. Oxon: Routledge.

Jewitt, C. 2014. Preface. In A. Archer and D. Newfield (eds.) Multimodal approaches to research and pedagogy: Recognition, resources and access. London and New York: Routledge. pp. xv-xvi.

Kastman Breuch, L. and S. Racine. 2000. Developing sound tutor training for online writing centres: Creating productive peer reviewers. Computers and Composition 17(3): 245-263.

Kell, C. 2006. Crossing the margins: Literacy, semiotics and the recontextualisation of meanings. In K. Pahl and J. Rowsell (eds.) Travel notes from the new literacies studies. Clevedon, Buffalo and Toronto: Multilingual Matters. pp. 147-172.

Kress, G. 2000. Multimodality. In B. Cope and M. Kalantzis (eds.) Multiliteracies. Literacy learning and the design of social futures. London and New York: Routledge. pp. 182-202.

Kress, G. 2010. A social semiotic approach to contemporary communication. London and New York: Routledge.

Kress, G. and S. Selander. 2012. Multimodal design, learning and cultures of recognition. Internet and Higher Education 15(4): 265-268.

Kress, G. and T. van Leeuwen. 2006. Reading images. The grammar of visual design. London: Routledge.

Lee, S. and R.G. Carpenter. 2013. The Routledge reader on writing centres and new media: Digital literacies in multimodal spaces. New York and London: Routledge.

Pearman, A. 2017. Supporting academic communication in writing centres in the digital age: Video composition in the context of a writing centre consultation. In S. Clarence and L. Dison (eds.) Writing centres in higher education: Working in and across the disciplines. Stellenbosch: Sun Media. pp. 177-192.

Prince, R. and A. Archer. 2014. Exploring academic voice in multimodal quantitative texts. Literacy and Numeracy Studies 22(1): 39-57.

Stein, P. 2008. Multimodal pedagogies in diverse classrooms: Representation, rights and resources. London and New York: Routledge. 
Thesen, L. 2014. Risk as productive: Working with dilemmas in the writing of research. In L. Thesen and L. Cooper (eds.) Risk in academic writing. Postgraduate students, their teachers and the making of knowledge. Bristol, Buffalo and Toronto: Multilingual Matters. pp. 1-24.

Weiss, R. 2014. Multimodality and medicine: Designing for social futures. In A. Archer and D. Newfield (eds.) Multimodal approaches to research and pedagogy: Recognition, resources and access. Oxon and New York: Routledge. pp. 153-173. 